\title{
Revitalisierung Kleinwasserkraftwerk Hollersbach durch Neubau von Druckrohrleitung und Krafthaus
}

\begin{abstract}
Zusammenfassung: Als das Kraftwerk Hollersbach in den Jahren 1947 bis 1949 gebaut wurde, war es in der Lage, den gesamten Oberpinzgau mit Strom zu versorgen. Heute reicht es gerade noch etwa 2200 Haushalte zur versorgen. Auf Grund der geringen Ausbauwassermenge ergibt sich ein erhebliches Ausbaupotenzial für die bestehende Anlage. Im Frühling des Jahres 2008 fasste die Salzburg AG daher den Entschluss, das Kleinwasserkraftwerk umzubauen. Die Modernisierung ermöglicht eine Ertragssteigerung auf rund 19 Mio. kWh jährlich, was beinah einer Verdreifachung der bisherigen Stromproduktion gleichkommt.
\end{abstract}

Revitalising the small Hollersbach power station by reconstructing the penstock and powerhouse

Summary: When the Hollersbach power station was built between 1947 and 1949, it was capable of supplying the whole Oberpinzgau district of the Salzach valley in the Province of Salzburg with electricity. Today it is just sufficient to cater for about 2200 homes. The fact that the plant was designed for a low rated discharge leaves considerable potential for development. In the spring of 2008, Salzburg AG decided to upgrade the small power station. Modernising this plant will increase the annual electricity generation to about 19 million $\mathrm{kWh}$, corresponding to almost three times its present production.

\section{Bestehende Anlage}

Das Kleinwasserkraftwerk nutzt das Wasser aus dem Hollersbach mit einem Einzugsgebiet von $67,3 \mathrm{~km}^{2}$ und des Bürgerbaches mit $2,4 \mathrm{~km}^{2}$.

Durch die Errichtung des 16,5 m hohen Schüttdammes Hollersbach entstand ein Tagesspeicher mit ca. $135.000 \mathrm{~m}^{3}$ Nutzinhalt. Der Staudamm hat eine Kronenlänge von $87 \mathrm{~m}$ und die luft- und wasserseitigen Böschungen sind 1:2 geneigt.

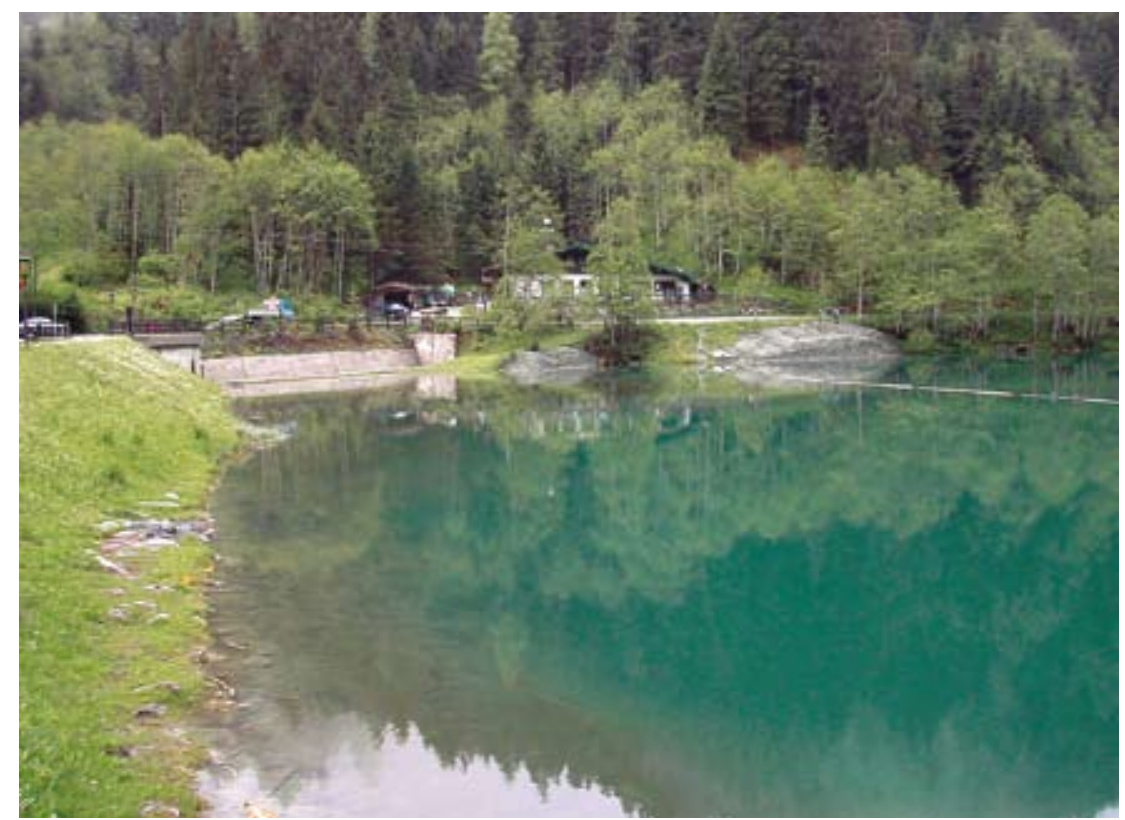

Abb. 1: Wasserfassung über Staudamm Hollersbach

Die Stauanlage verfügt über drei Hochwasserentlastungsanlagen, da sich im Lauf der Jahre die sicherheitstechnischen Anforderungen entscheidend erhöhten. Eine wesentliche Rolle spielte der Staudamm mit einem Unholzrückhalt für den Hochwasserschutz des Ortes Hollersbach.

Die Förderfähigkeit des Grundablasses beträgt beim derzeitigen Stauziel auf $879,45 \mathrm{~m}$ ü. A. ca. $72 \mathrm{~m}^{3} / \mathrm{s}$. Über den 104,5 m langen Grundablassstollen mit einem Querschnitt von 9,1 $\mathrm{m}^{2}$ wird das Wasser wieder in den Hollersbach geleitet.

Das Triebwasser wird linksseitig über einen Feinrechen mit Rechenreinigungsmaschine über einen $555 \mathrm{~m}$ langen Druckstollen mit $2,5 \mathrm{~m}^{2}$ Fläche zum Schachtwasserschloss geleitet. Vom Wasserschloss führt der Triebwasserweg über einen 45,9 m langen Rohrstollen mit einem Stahlrohr DN 1000 in die Apparatekammer, in der ein Dehn- und Ausbaustück eingebaut wurde, und über die ca. $435 \mathrm{~m}$ lange Druckrohrleitung DN 1000 zum Krafthaus.

Im Krafthaus wird über zwei Francisturbinen mit einer Leistung von $700 \mathrm{~kW}$ und je $1,3 \mathrm{~m}^{3} / \mathrm{s}$ Ausbauwassermenge das Wasserdargebot abgearbeitet. Über den 324 m langen Unterwasserkanal, von dem eine Strecke von $219 \mathrm{~m}$ als Betonkanal DN 1500 ausgeführt wurde, wird das Triebwasser in den Hollersbach zurück gegeben. Die Jahreserzeugung des bestehenden Kleinwasserkraftwerkes beträgt rund 7,5 Mio. kWh.

\section{Studien zur Revitalisierung}

Die Prüfung verschiedener Varianten wie beispielsweise der Ertüchtigung des bestehen Triebwasserstollens mit Vergrößerung des Wasserschlosses sowie Neubau der Druckrohrleitung ergab, dass ein neuer Triebwasserweg mit einer Verlegung des Krafthauses an das Ufer der Salzach die wirtschaftlichste Variante darstellt. Entscheidend war vor allem, dass der Einbau eines größeren Maschinensatzes in das alte Krafthaus sehr zeitaufwändig, kompliziert und unwirtschaftlich gewesen wäre. Weiters könnte der Unterwasserkanal nur eine Ausbauwassermenge von ca. $5,0 \mathrm{~m}^{3} / \mathrm{s}$ abführen. 


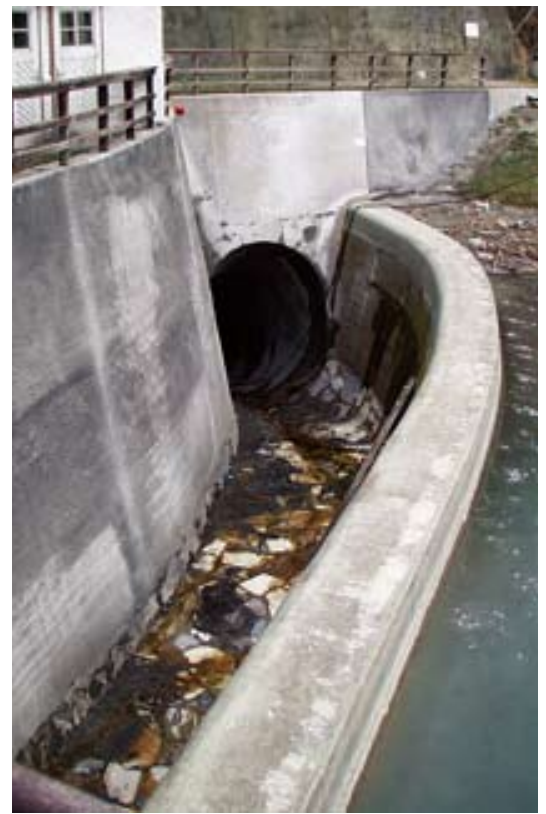

Abb. 2: Linksseitiger Hochwasserentlastungstrog

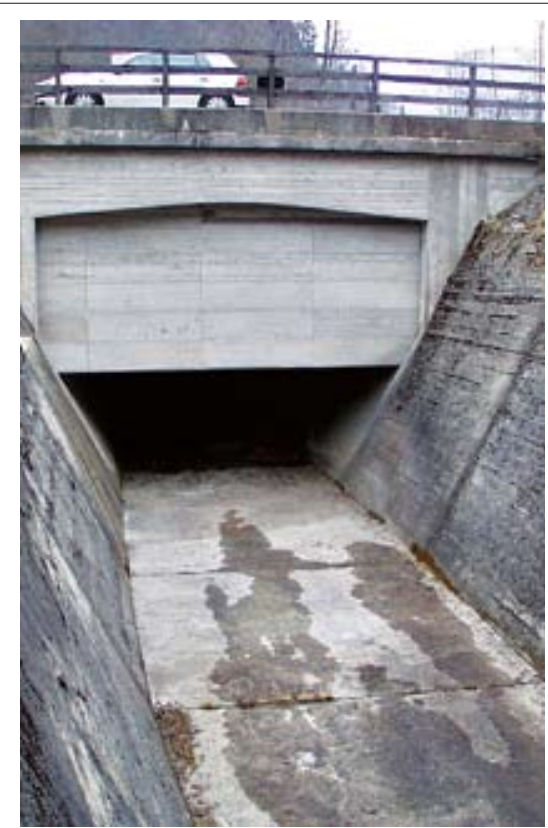

Abb. 3: Rechtseitige Hochwasserentlastungstrog

Abb. 4: Hochwasserentlastungsgerinne über Dammkrone

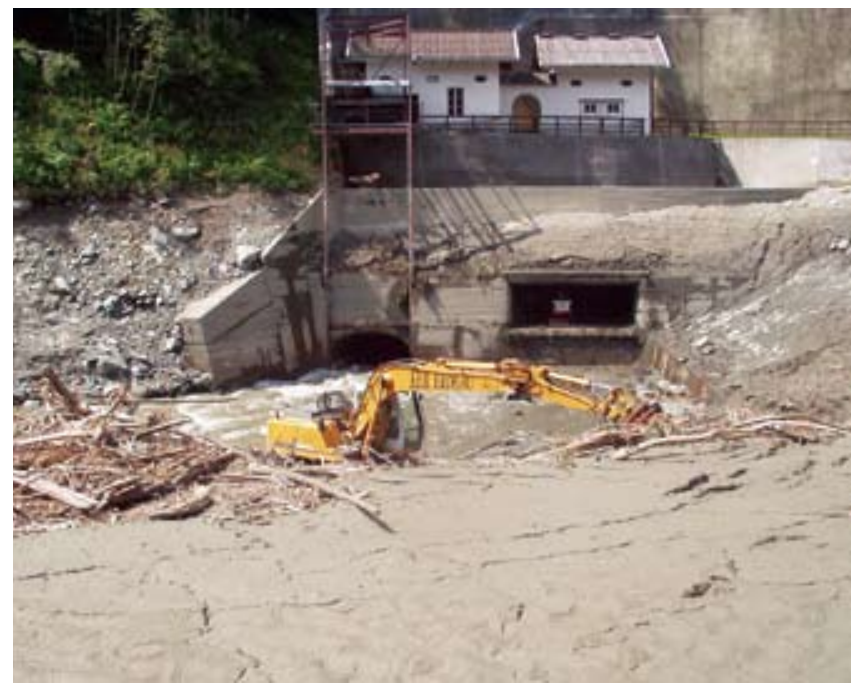

Nach Vorliegen der Variantenstudien wurde im März 2008 von der Salzburg AG der Beschluss gefasst, die Anlage $\mathrm{zu}$ revitalisieren und alle behördlichen Genehmigungen für den Ausbau des bestehenden Kraftwerks zu erwirken. Aufgrund des von der Salzburg AG Bereich Sonderprojekte/Bautechnik (Projektleitung Dipl.-Ing. Alfred Wolf) erstellten Vorprojektes konnte die Detailprojektierung für die Ertüchtigung des Staudammes sowie den Neubau von Krafthaus und Druckrohrleitung vergeben werden.

Ziel des Ausbauvorhabens ist es, durch eine Erhöhung der Ausbauwassermenge von $2,6 \mathrm{~m}^{3} / \mathrm{s}$ auf $7,5 \mathrm{~m}^{3} / \mathrm{s}$ und durch die Verlegung des KrafthausStandortes aus dem Ortszentrum von Hollersbach an das Salzachufer, die Anlage $\mathrm{zu}$ optimieren. Es werden alle wesentlichen Anlageteile, mit Ausnahme des anzupassenden Staudammes, neu errichtet. Die Fallhöhe wird um rund $15 \%$ auf $84 \mathrm{~m}$ erhöht und das Regelarbeitsvermögen von derzeit 7,5 Mio. kWh um rund $250 \%$ auf zukünftig 19 Mio. kWh gesteigert. Als besonderer Vorteil dieser Lösung ist zu erwähnen, dass das alte Kraftwerk bis auf die Zeit der Errichtung des neuen Einlaufbauwerkes seinen Betrieb aufrecht erhalten kann. Im Zuge des neuen Ausbaus wird es auch erforderlich, den alten Triebwasserweg still zu legen und das Krafthaus ab zu tragen. Die Parzelle des Krafthauses wird von der Gemeinde Hollersbach künftig für die Gestaltung des Ortskernes verwendet werden. Der Unterwasserkanal wird ebenfalls von der Gemeinde Hollersbach übernommen und als Retensionsbecken in das Regenwasserentsorgungssystem eingebunden.

Die wasser-, naturschutz-, forstund elektrizitätsrechtlichen Bewilligungsverfahren fanden im Dezember 2008 statt und alle Bescheide lagen Anfang Februar 2009 vor.

\section{Hydrologie und Pflichtwasser- abgabe}

Die Tabelle 1 gibt die monatlichen Mittelwasserabflüsse $\mathrm{m}^{3} / \mathrm{s}$ an. [B. Staudinger, Hydrografischer Dienst, 2008]

Jahresmittelwasser:

$\mathrm{MQ}=3,2 \mathrm{~m}^{3} / \mathrm{s}$

Niederwasser:

MJNQT $=270,0 \mathrm{l} / \mathrm{s}$

$\mathrm{NQT}=100,0 \mathrm{l} / \mathrm{s}$ 


\begin{tabular}{|c|c|c|c|c|c|c|c|c|c|c|c|c|}
\hline Monat & 1 & II & III & IV & $\mathrm{V}$ & $\mathrm{VI}$ & $\mathrm{VII}$ & VIII & IX & $x$ & $\mathrm{XI}$ & XII \\
\hline Abfluss & 0,7 & 0,5 & 0,8 & 1,7 & 5,4 & 6,7 & 7.7 & 5,7 & 3,7 & 2,7 & 1,7 & 1,0 \\
\hline
\end{tabular}

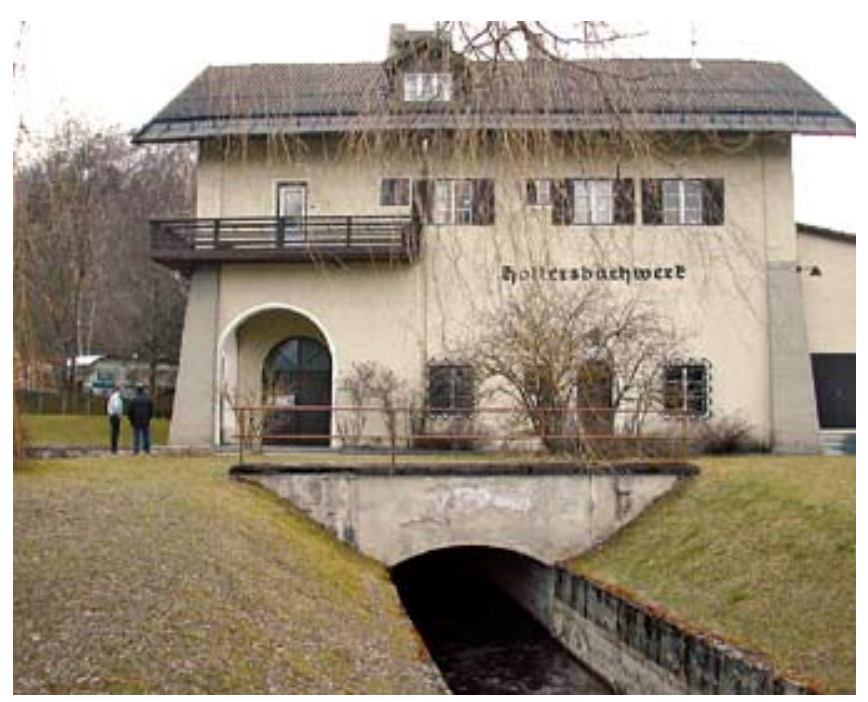

Abb. 6: Bestehendes Krafthaus Hollers-

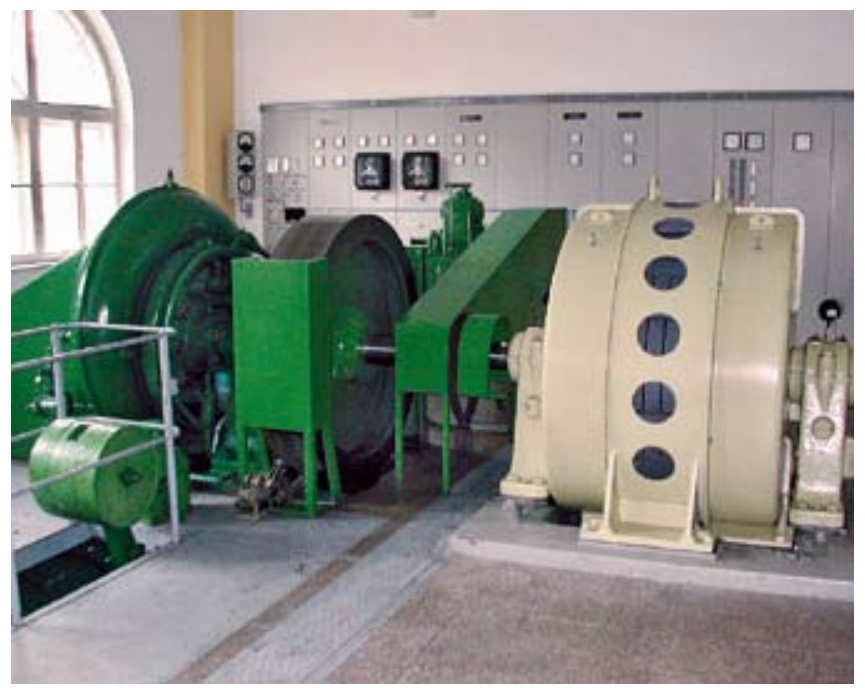

Abb. 7: Maschinensatz im bestehenden Krafthaus

Hochwasserabflusswerte:

HQ5000 = $260 \mathrm{~m}^{3} / \mathrm{s}$ (RHHQ laut HZB)

$\mathrm{BHQ}^{*}=275 \mathrm{~m}^{3} / \mathrm{s}$

$\mathrm{SHQ}^{*}=358 \mathrm{~m}^{3} / \mathrm{s}$

Die Abgabe einer den Signifikanzkriterien der „Ist-Bestandserhebung“ des BMLFUW entsprechenden Pflichtwassermenge ist ab dem Damm des Speichers vorgesehen:

$$
\begin{aligned}
& M Q_{R W} \geq M J N Q_{T(\text { nat })} \\
& N Q_{T, R W} \geq N Q_{T(\text { nat })}
\end{aligned}
$$

Das Restwasser ergibt sich aus dem bescheidgemäß vorgeschriebenen Dotier- wasser und dem nicht eingezogenen, natürlichen Überwasser:

$Q_{R W}=Q_{\text {Dot }}+Q_{\text {nat. Überw. }}$

Der bisher zur Nutzung beigeleitete Bürgerbach mit etwa $2,4 \mathrm{~km}^{2}$, wird hingegen zukünftig nicht mehr gefasst, sondern ganzjährig als Restwasser abgegeben. Das Gesamteinzugsgebiet umfasst weiterhin $69,7 \mathrm{~km}^{2}$.

\section{Technische Details}

Revitalisierung und neue Anlage

Der Damm Hollersbach soll um 1,55 m auf 18 Meter erhöht werden, wobei gleichzei- tig eine Anpassung an die neuen Hochwasserschutzrichtlinien erfolgen wird. Die Innenkerndichtung wird durch einen Dichtschirm ergänzt und das Speichervolumen somit zukünftig auf $175.000 \mathrm{~m}^{3} \mathrm{er}-$ höht.

Die Triebwasserentnahme erfolgt über Stahlbetonrohre mit einem Außendurchmesser von $2500 \mathrm{~mm}$ die vom Schützenhaus ca. $35 \mathrm{~m}$ durch den Dammkörper und den Dichtschirm gepresst werden. Die neue Druckrohrleitung, eine GFUP-Rohrleitung DN $1.800 \mathrm{~mm}$ mit einer Gesamtlänge von $1.620 \mathrm{~m}$, quert zukünftig ca. $50 \mathrm{~m}$ nach dem Schützenhaus den Bürgerbach. Anschließend verläuft die Leitung überwiegend parallel zur Genossenschaftsstraße an der orografisch rechten Talseite bis zur Hollersbachbrücke. Nach Querung der Landesstraße im Ortszentrum und weiteren $500 \mathrm{~m}$ wird die Bundesstraße mittels Rohrdurchpressung gequert und die Trasse führt über Wiesengrundstücke zum Krafthaus am Salzachufer. Die Druckrohrleitung DN 1800 wird ausgehend vom Einlaufbauwerk bis zum Messschacht in Stahl ausgeführt Der Materialwechsel auf GFUP-Rohre erfolgt unmittelbar nach Durchführung der Rohrleitung durch den Messschacht. Im Messschacht wird eine außen aufgeschnallte Durchflussmessung mittels Ultraschall situiert, um die Differenzmengenmessung mit einer weiteren beim Krafthaus situierten Messung bewerkstelligen zu können. Darüber hinaus wird in diesem Messschacht die Durchflussmessung für die Restwasserleitung DN500 eingebaut. Im Bereich der Trasse der Druckrohrleitung sind verschiedenste Einbauten wie die Trinkwasserleitung der Wassergenossenschaft Hollersbach, der Abwasserkanal der Gemeinde, Versorgungskabel der Post und Telekom sowie Strom- und Energiekabel der Salzburg AG vorhanden, die zum Teil umgelegt werden müssen.

Das neue Krafthaus liegt zwischen Bundesstraße und Salzach, nordöstlich der ehemaligen Kläranlage und dem heutigen Recyclinghof der Gemeinde Hollersbach. Die Zufahrt ist ausgehend von der Bundesstraße über den bestehenden Treppelweg möglich. Der geplante Krafthausstandort wird durch die $110 \mathrm{kV}$ und $380 \mathrm{kV}$ Freileitung überspannt. Die Gründung des 


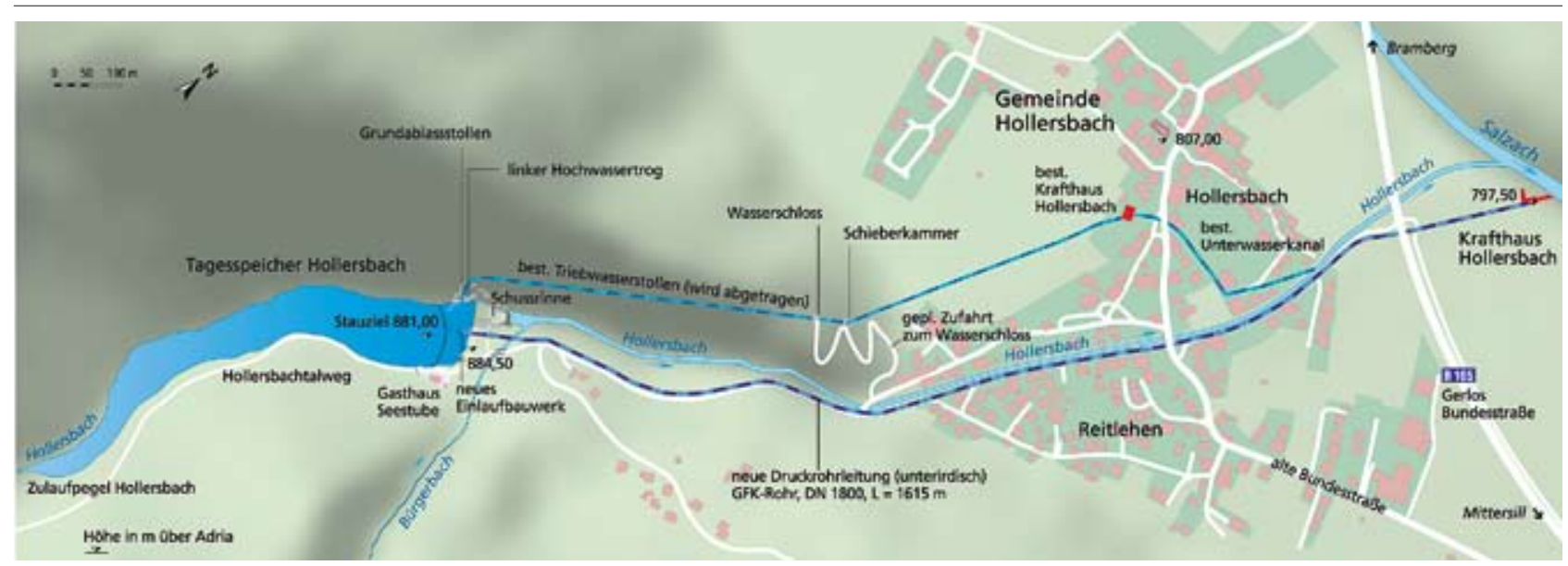

Abb. 8: Übersichtslageplan alter und neuer Triebwasserweg

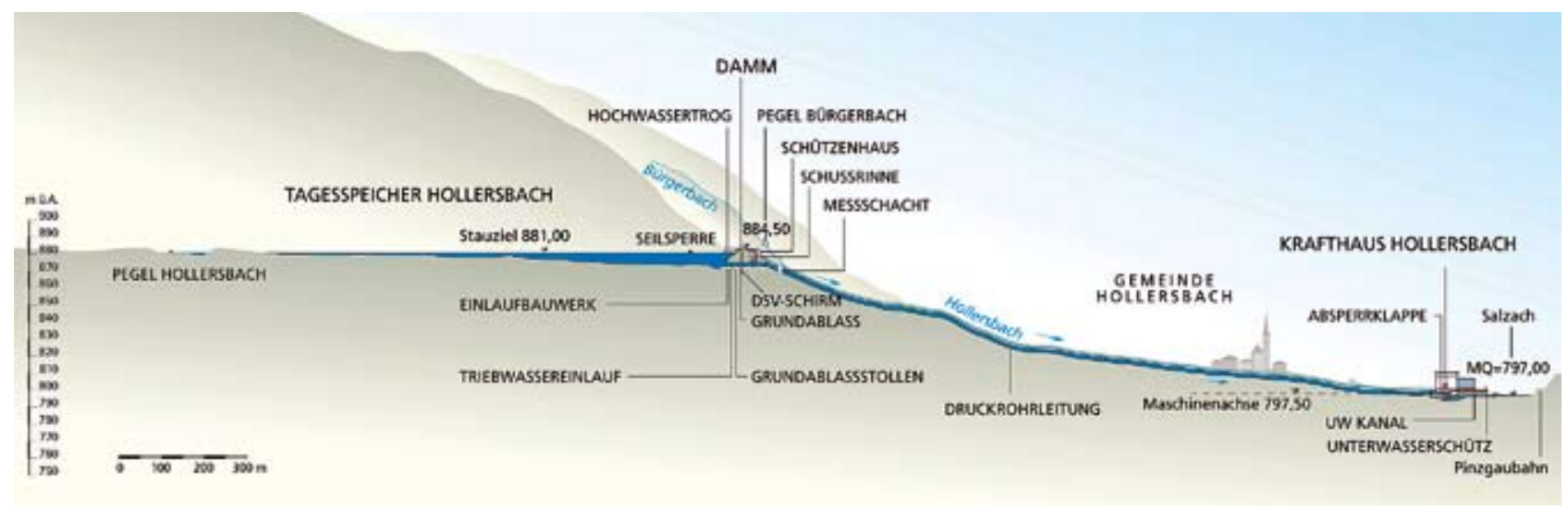

Abb. 9: Längenschnitt neue Anlage und hydraulisches Schema

Krafthauses erfolgt in anstehenden Salzach-Schottern mit einer Flachgründung. Die Maschinenhalle mit den Außenabmessungen 13,35 x 9,70 m sowie einer lichten Höhe von 6,65 m erstreckt sich im wesentlichem in Richtung Südosten. An die Maschinenhalle angebaut ist ein Werkstättenraum mit den Innenabmessungen $4,70 \times 6,10 \mathrm{~m}$. Der Abschnitt des Büro- und Aufenthaltsraumes, Sanitäranlagen, sowie in weiterer Folge $30 \mathrm{KV}$-Schaltanlage und Traforaum bilden eine L-förmigen Baukörper mit der Maschinenhalle. Im Krafthaus kommt eine Francis-Turbine samt Generator in vertikaler Bauweise zur Aufstellung. Des Weiteren sind im Krafthaus die Generatorzellen, Umspanner, eine $30 \mathrm{kV}$-Schaltanlage und der Eigenbedarfstrafo, die Maschinenautomatiken, die Schutzeinrichtungen, der Leitstand, die Batterien und Gleichrichter, sowie diverse maschinelle und elektrische $\mathrm{Ne}$ beneinrichtungen vorzufinden. Die erzeugte Energie wird auf $30 \mathrm{kV}$ transformiert und ins $30 \mathrm{kV}$-Verteilernetz der Salzburg AG eingespeist. Ferner ist im
Krafthaus ein Montagekran mit der nötigen Tragkraft für die Montage der Turbinen und Generatoren vorgesehen.

\section{Turbine}

Bauart: Francis mit vertikaler Achse

Nenndurchfluss: $7,5 \mathrm{~m}^{3} / \mathrm{s}$

Nennleistung: $5353 \mathrm{~kW}$

Nenndrehzahl: $600 \mathrm{U} / \mathrm{min}$

Wirkungsgrad: ca. 92,5\%

Die Regelung der Turbine erfolgt über den Leitapparat mittels elektrohydraulischer Turbinenregler.

\section{Generatoren}

Nenneistung: 6200 kVA

Nennfrequenz: $50 \mathrm{~Hz}$

$\cos \varphi: 0,85$

Nennspannung: $6300 \mathrm{~V}+/-5 \%$

Der Generator ist in vertikaler Bauweise konzipiert und direkt mit der Turbinenwelle gekuppelt. Die Erregung erfolgt mittels auf der Welle sitzender Erregermaschine und rotierenden Dioden. Der Generator ist luftgekühlt und die Luft wird in Luft-Wasser-Wärmetauschern im geschlossenen Kühlkreislauf rückgekühlt.
30 kV-SF6 Schaltanlage: Die an den Generatorklemmen abgegebene Energie wird mittels Schienenverbindungen und Kabeln zu den 6,3 kV-Zellen geführt und von dort ebenfalls mittels Schienen bzw. Kabeln zum Umspanner, welcher in einer eigenen Box situiert ist, geführt.

In der $30 \mathrm{kV}$-Anlage sind Stromund Spannungswandler für Schutz, Messung, Zählung und Regelung sowie Erdschlusswiderstände, Erregertrafos und Trennschalter untergebracht. Die Schaltanlage besteht aus 6 Zellen und ist als Innenraumschaltanlage in SF6-Bauweise ausgeführt. Die Verbindungen nach außen bzw. zu den Trafos erfolgen über Kabel. Die Anlage ist sowohl vor Ort als auch fernsteuerbar.

Elektrische Kennwerte:

höchste Spannung für Betriebsmittel: $36 \mathrm{kV}$ Sammelschienenstrom: $630 \mathrm{~A}$

Blocktransformator: Die Maschinenspannung bzw. Schaltanlagenspannung von $6,3 \mathrm{kV}$ wird über den Blocktransformator auf die Netzspannung von $30 \mathrm{kV}$ transformiert. 


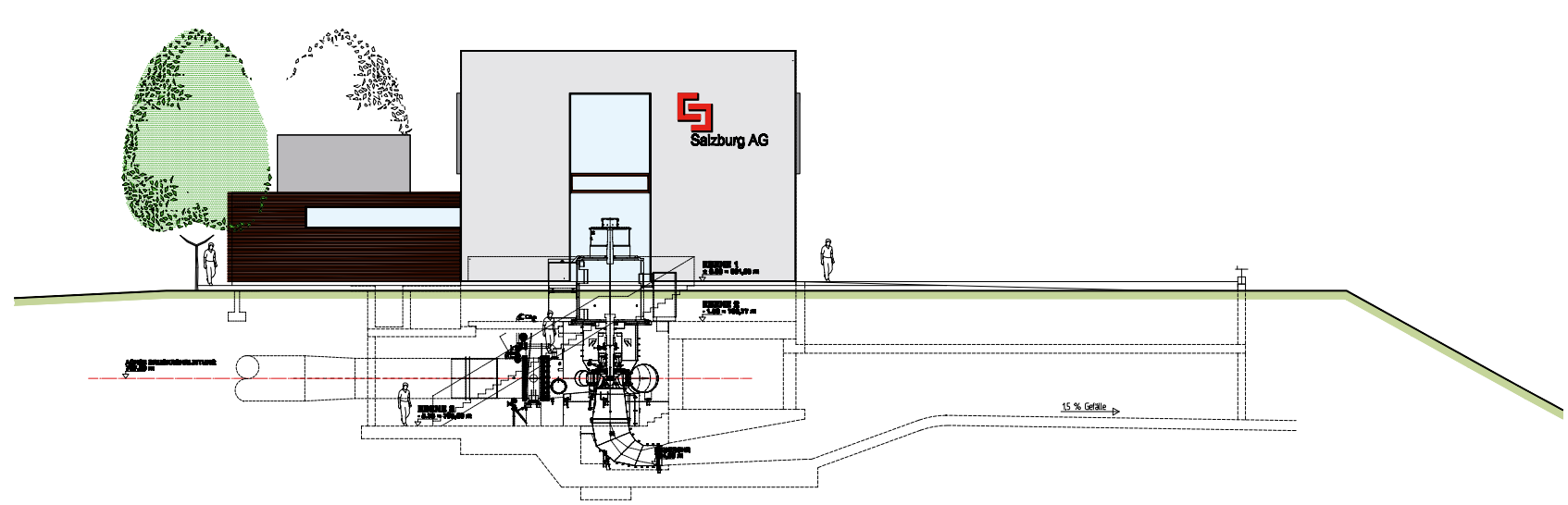

Abb. 10: Süd-Ostansicht neues Krafthaus

Technische Daten Blocktrafo:

Nennleistung: $6.300 \mathrm{kVA}$

Übersetzungsverhältnis: 6300/30000 V

Gesamtgewicht: ca. 14,5 t

Der Blocktrafo befindet sich im unmittelbaren Bereich des Krafthauses und ist von außen zugänglich.

Batterie und Gleichrichter: Zur Versorgung der Automatik, für die Notund Signallichtversorgung, sowie für Fernmelde- und Fernwirkeinrichtungen etc. dient eine $24 \mathrm{~V}$ Batterie. Die Batterien und Gleichrichter kommen im Erdgeschoß (Generatorniveau) zur Aufstellung.

Steuerung des Maschinensatzes und der Schaltanlage: Das Kraftwerk ist für unbesetzten Betrieb konzipiert. Für die Steuerung des Maschinensatzes ist im Krafthaus ein Maschinenleitstand vorgesehen. Die Steuerung der Anlagen erfolgt durch die Einsatzleitstelle in Salzburg oder durch die Kraftwerksgruppe Oberpinzgau (Warte Kraftwerk Wald und Heimwarte, Bereitschaftsdienst).

Fernwirkeinrichtung: Die nötigen Einrichtungen für Fernwirk- und Fernmeldezwecke sind im Leitstand untergebracht.

Schutz und Überwachungseinrichtungen: Diese sind in elektronisch/digitaler Bauweise vorgesehen. Sie schützen die Hauptkomponenten des Kraftwerkes wie Maschinensatz, Transformator, $30 \mathrm{kV}$ Schaltanlage vor den Auswirkungen von Fehlern und Fehlbedienungen. Sämtli- che Gefahrenmeldungen aus den einzelnen Anlagenteilen werden in einem zentralen Leittechnikgerät erfasst.

Krane: Zur Montage der Turbine und Generatorteile bzw. deren Komponenten ist in der Maschinenhalle ein Kran mit einer Tragkraft von 12,5t vorgesehen.

Kühlwasseranlage und Entwässerungsanlage: Für die Kühlung der erwärmten Generatorenkühlluft und des Lageröles wird Kühlwasser benötigt. Dieses wird mit Kühlwasserpumpen aus dem Wärmetauscher im Unterwasser angesaugt und dem Kühlkreislauf zugeführt. Die Kühlwasserpumpe, Filter und Absperrorgane sind am Turbinenboden installiert.

Energieableitung: Die $30 \mathrm{kV}$ Energieableitung erfolgt zur TRST Gewerbe Ost in Hollersbach.

Unterwasserkanal: Der Unterwasserkanal zur Salzach liegt im Norden der Maschinenhalle und weist ausgehend vom Saugrohrende eine Breite von 5,0 m auf. Unmittelbar an der Außenwand der Maschinenhalle wird ein Einstieg in den Unterwasserkanal hergestellt und ein Unterwasserschütz angeordnet damit bei höherer Wasserführung in der Salzach Arbeiten an der Turbine möglich sind.

Stärkung der heimischen Wirtschaft: 60 Jahre nach Errichtung des Kraftwerks Hollersbach plant die Salzburg AG nun die Modernisierung der Anlage.
Durch die umfangreichen Maßnahmen ist eine beachtliche Effizienzsteigerung des bisherigen Kleinwasserkraftwerkes möglich. Darüber hinaus stellt in Zeiten wie diesen der Ausbau des Kraftwerkes Hollersbach mit einem voraussichtlichen $\mathrm{Ge}$ samtprojektkosten von rund 16,5 Mio. Euro sicherlich einen Beitrag zur Stärkung der heimischen Wirtschaft dar und leistet einen bedeutenden Beitrag zur regionalen Versorgungssicherheit.

\section{Zusammenfassung}

Geht alles weiter nach Plan, könnte schon im August 2009 mit den Bauarbeiten begonnen werden. Eine Fertigstellung wird für Juli 2010 avisiert. Dann kann das volle Potenzial des Hollersbaches für die Erzeugung von Strom aus erneuerbarer Wasserkraft für die Versorgung von ca. 5000 Haushalten genutzt werden.

Korrespondenz:

Salzburg AG, Bereich Sonderprojekte, Center Bautechnik Bayerhamerstrasse 16 A-5020 Salzburg

Tel:+43/662/8884-2077

Fax:+43/662/8884-170-2077

E-Mail: alfred.wolf@salzburg-ag.at

\section{LITERATUR}

Seiwald S (2008) Vollausbau nach 60 Jahren, ENERGIE + KOMMUNALin zek ZUKUNFTS- 